\title{
NOTE \\ Internal Medicine \\ Long-term management of a dog with idiopathic megaesophagus and recurrent aspiration pneumonia by use of an indwelling esophagostomy tube for suction of esophageal content and esophagogastric tube feeding
}

\author{
Yuka KANEMOTO ${ }^{1,2)}$, Kenjiro FUKUSHIMA ${ }^{1)^{*}}$, Hideyuki KANEMOTO1), \\ Koichi OHNO ${ }^{1)}$ and Hajime TSUJMOTO') \\ 1)Department of Veterinary Internal Medicine, Graduate School of Agricultural and Life Sciences, The University \\ of Tokyo, 1-1-1 Yayoi, Bunkyo-ku, Tokyo 113-8657, Japan \\ 2)Japan Animal Referral Medical Center of NAGOYA, 1-602 Konosu, Tenpaku-ku, Nagoya, Aichi 468-0003, Japan
}

J. Vet. Med. Sci.

79(1): 188-191, 2017

doi: 10.1292/jvms.16-0374

Received: 15 July 2016

Accepted: 31 October 2016

Published online in J-STAGE:

17 November 2016
ABSTRACT. A 10-year-old neutered male Labrador Retriever dog was diagnosed with idiopathic megaesophagus. Despite receiving conventional treatments including elevated feeding, the dog showed repeated regurgitation and aspiration pneumonia, consequently developing weight loss and severe malnutrition. For the purpose of controlling regurgitation, an esophagostomy tube was placed for draining the esophageal fluid. Additionally, an esophagogastric tube was placed for nutritional support. After tube placement, the average frequency of regurgitation was reduced from 2.4 times a day to 0.1 times a day. The nutritional state of the dog improved gradually, and the body weight increased from 18.5 to $27.9 \mathrm{~kg}$. The dog died on day 951, and necropsy revealed a gastric ulcer ( $2.5 \mathrm{~cm}$ in diameter), presumably esophagostomy tube-induced injury. This case report suggests that patients with idiopathic megaesophagus and persistent regurgitation might benefit from esophageal drainage through an esophagostomy tube.

KEY WORDS: aspiration pneumonia, canine, esophagostomy tube, megaesophagus

Megaesophagus (ME) is a disorder characterized by decreased or absent esophageal motility that results in a diffuse dilation of the esophagus $[13,14]$. Depending on the cause, ME is classified into primary ME, which is idiopathic, or secondary ME, which occurs in conjunction with other diseases including myasthenia gravis, hypoadrenocorticism, dysautonomia, polyradiculoneuritis, hypothyroidism, polymyopathies and esophageal cancer [1, 2, 4, 6, 9-11, 15]. A recent study reported that most cases of canine ME were idiopathic (IME) (76\%), and the rest were secondary ME (24\%), mainly involving myasthenia gravis [7]. While the treatment for secondary ME is dependent on the underlying disease, there is no curative treatment for IME. Treatment for IME mainly consists of symptomatic treatment, such as upright feeding [12, 13]. However, patients with severe ME may develop weight loss, weakness and often complicated aspiration pneumonia (AP) [3,5]. The prognosis of ME is poor, and the median survival time has been reported to be 90 days in dogs [7]. The prognosis is especially poor in ME with complicating AP, and their median survival time is reported as 16 days [7].

A gastrostomy tube is one of the options for nutritional support in dogs with ME. However, regurgitation from the dilated esophagus is sometimes uncontrollable, consequently developing AP. Additionally, the gastrostomy tube is associated with a potential risk of peritonitis, especially in cases with malnutrition. An esophagostomy tube is another method to provide nutrition in dogs and cats. However, the tube placement is not suitable for patients with ME, since food and fluid administered to the esophagus would stay for a prolonged time and increase the risk of AP. In this case report, we have described long-term management of IME with complicating AP using an esophagostomy tube for draining esophageal fluid and a transesophagealgastric tube for nutritional support.

A 10-year-old, neutered male Labrador Retriever was referred to the Veterinary Medical Center of the University of Tokyo (VMC-UT) with a chief complaint of chronic regurgitation. Thoracic radiographs revealed generalized ME. On blood examination, total T4, free T4, acetylcholine-receptor-antibody values, creatinine phosphokinase activity and electrolytes were within normal range. As no significant abnormalities were observed on abdominal ultrasonography, endoscopic examination revealed

*Correspondence to: Fukushima K., Department of Veterinary Internal Medicine, Graduate School of Agricultural and Life Sciences, The University of Tokyo, 1-1-1 Yayoi, Bunkyo-ku, Tokyo 113-8657, Japan. e-mail: akenjiro@mail.ecc.u-tokyo.ac.jp

(C2017 The Japanese Society of Veterinary Science 
megaesophagus and mild chronic gastroenteritis. Since there was no macroscopic finding related to esophagitis, biopsy sample was not obtained from esophagus. Since the underlying disease had been excluded, the dog was diagnosed as having IME. Although the dog underwent symptomatic treatments, such as upright feeding, for 20-30 min every post meals and administration of prokinetics (mosapride, $1 \mathrm{mg} / \mathrm{kg}$, twice daily), response to these treatments was poor. Information about regurgitation frequency was collected from the owner at every revisit. The average regurgitation frequency was calculated by determining the total number of regurgitation episodes between the previous to the present visit divided by the number of days between the two visits. Over this period, the dog regurgitated 1-10 times a day (average frequency of regurgitation: 2.4 times a day). Therefore, the dog repeated AP (diagnosed by radiography), and C-reactive protein (CRP) level was sustainably high (average: $4.6 \mathrm{mg} / \mathrm{d} l$ ). As a result, from malnutrition and exhaustion, the dog's body weight gradually reduced from $31.1 \mathrm{~kg}$ to $18.5 \mathrm{~kg}$. Although we suggested placing the gastrostomy tube for nutritional support, the owner was reluctant to pursue tube feeding at this point. On day 504, the dog presented to VMC-UT with recumbent state. The dog showed severe lethargy, weakness, labored respiration and hypothermia $\left(36.3^{\circ} \mathrm{C}\right)$. Blood examination revealed elevated CRP level $(19.5 \mathrm{mg} / \mathrm{d} l$; reference range, $<0.7 \mathrm{mg} / \mathrm{d} l)$ and blood urea nitrogen (BUN) level ( $82.8 \mathrm{mg} / \mathrm{d} l$; reference range, 9.2 to 29.2$)$, while creatinine concentration was within normal range $(0.7 \mathrm{mg} / \mathrm{d} l)$. Urinary specific gravity (USG) was 1.037 , and therefore, it is considered prerenal azotemia because of dehydration. On thoracic radiographs, radiopaque alveolar pattern was observed on right and left cranial lobes, and right middle lobe, indicating AP.

At this visit, we planned to place the esophagostomy tube (E-tube) for draining esophageal fluid to prevent esophageal regurgitation. Additionally, we planned to place the transesophageal-gastric tube (EG-tube) for stable nutritional support. The gastrostomy tube was not selected this time, because of the severely malnourished state of the dog and the risk of life threatening complications related with this procedure, such as peritonitis. With treatment (antibiotics and fluid therapy), the AP resolved within 9 days of hospitalization. On day 9 after admission (day 513), E-tube and EG-tube placement were performed under general anesthesia.

E-tube (Gastrointestinal catheter, 20Fr., TERUMO CO., Tokyo, Japan) was prepared with several side holes that were made to enable aspiration from wide ranges and reduce the risk of clogging. Subsequently, EG-tube placement (PEG-kit, 20Fr., MILA INTERNATIONAL. Inc., Florence, KY, U.S.A.) was performed. The tip of the EG-tube was brought with endoscopy and located in the area of gastric fundus $3 \mathrm{~cm}$ past the cardia (Fig. 1A and 1B). Each tube was fixed by Chinese finger trap suture procedure.

From the E-tube, esophageal fluid and gas were removed effectively (10-380 $\mathrm{m} /$ /day and 15-1,500 $\mathrm{m} l /$ day, respectively), and regurgitation was not observed during hospitalization. Resting energy requirement was achieved after 4 days from EGtube placement. The owner had been provided instructions to perform drainage and feeding three times daily, and the dog was discharged 15 days after admission. After discharge, esophageal fluid was removed (300-750 ml daily), and the dog did not show regurgitation and complicated AP unless E-tube clogging occurred. The average frequency of regurgitation after E-tube placement was reduced to 0.1 times a day during the follow-up period. As the nutritional state gradually improved with feeding from the EG-tube, the dog became ambulatory in stages during one month after admission (day 540). Body weight increased appreciably from 18.5 to $27.9 \mathrm{~kg}$ over 14 months (Fig. 2). Despite the placement of the 20 Fr. EG-tube from esophagus to stomach trans-cardia, reflux esophagitis was not observed on endoscopy performed to replace E-tube (day 836). The E-tube was easily and safely managed by the owner at home.

There were several complications related to this treatment. The most frequently observed complication was E-tube clogging. Minor or transient E-tube clogging was often observed during the treatment period. Severe E-tube clogging occurred 2 times (days 693 and 836), and a need to replace the E-tube occurred twice (the first E-tube which was made of polyvinyl chloride was hardened by gastric acid). In addition, the dog developed chronic kidney disease (CKD) on day 690. Because of sustained esophageal fluid loss and CKD, the dog exhibited hyponatremia (sodium concentration was 129 (meq/L)) on day 799, although it was managed by the administration of subcutaneous saline infusion and electrolyte replenisher through EG-tube. On day 836 when the second severe E-tube clogging occurred, we performed endoscopy to replace the E-tube and found the polyp at the cardia, and histopathological diagnosis of the polyp was mucosal hyperplasia. Since this lesion was found at the cardia where the tip of the EG-tube made contact continuously, the tip of the EG-tube was advanced few $\mathrm{cm}$ further from cardia. After that, the dog presented vomiting on day 938. Mild anemia (Hct 31\%), elevated CRP $(4.0 \mathrm{mg} / \mathrm{d} l$ ) and pancreatic lipase activity (635 U/l; reference range, $<160 \mathrm{U} / l$ ) were observed on blood examination. Ultrasound revealed dilated stomach. At that time, since acute pancreatitis was suspected, the dog was hospitalized for the fluid therapy and other symptomatic treatments. Although the anemia (Hct 29\%) and elevated CRP level $(12.5 \mathrm{mg} / \mathrm{d} l)$ pursued, general condition was improved and pancreatic lipase activity decreased to normal range. As the dog was discharged on day 443, it died at home on day 951. Necropsy revealed severe gastric ulcer on the gastric body (circular in shape and $2.5 \mathrm{~cm}$ in diameter) as a result of the EG-tube induced injury. The ulcer was extended to the muscular layer of the gastric wall with severe neutrophils infiltration. Other findings on necropsy included esophageal muscular atrophy, severe fibrosis in the kidney and multifocal granuloma in the lung (as a result of repeated aspiration pneumonia). Esophageal inflammation was not observed.

IME is known as a disease with poor prognosis, especially in cases with complicating AP [7]. Conventional treatments including upright feeding do not reduce the frequency of regurgitation and the risk of AP. In the present report, we showed that drainage of esophageal fluid by using an E-tube effectively decreased the frequency of regurgitation (2.4 times/day-0.1 times/day). Additionally, esophageal drainage can be performed by the owner easily and safely at home. Recently, it has been reported that the placement of esophageal drainage tube resulted in successful management of regurgitation and aspiration pneumonia in the 4 dogs with IME [8]. This procedure must be promising treatment option for the dogs with IME complicating AP. Since the tube clogged several times in the present study, it is necessary to consider the size and number of side holes. Since the first E-tube which was 

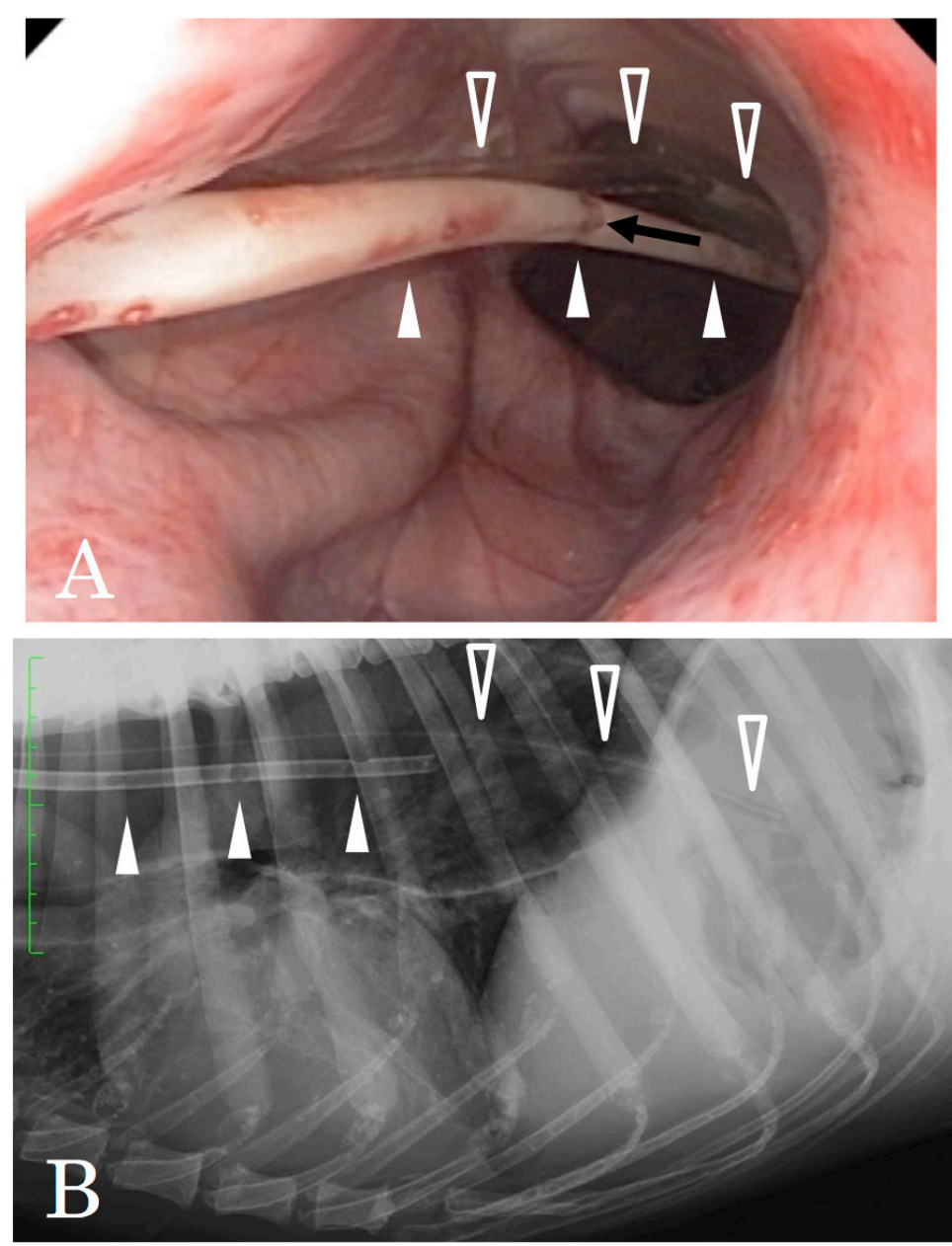

Fig. 1. A: Endoscopic view of the E-tube (white-filled triangle) and EG-tube (white-unfilled triangle). An aspect of the E-tube and several side holes (black arrow) were made. B: Radiographic view of the E-tube and EG-tube.

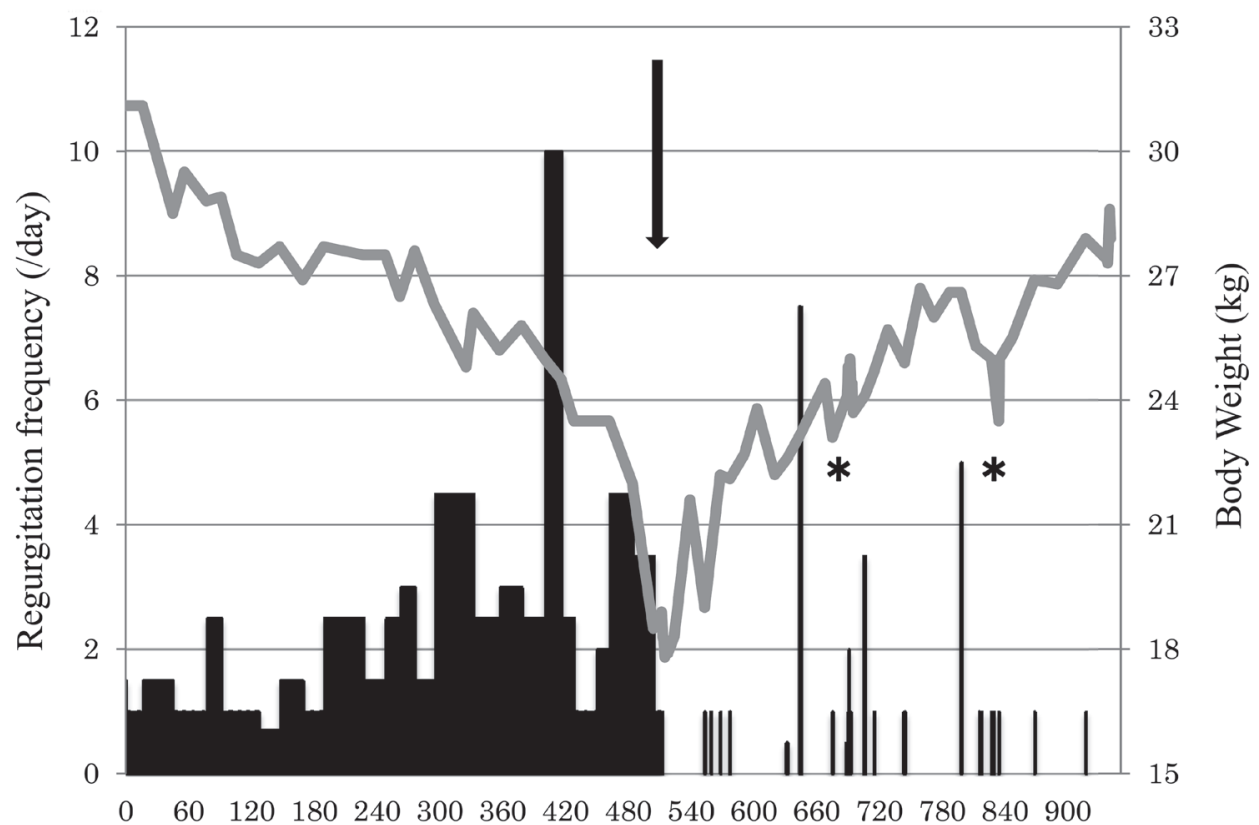

Fig. 2. Line graph (gray scale) shows the dog's body weight transition. Vertical bar graph (black scale) shows regurgitation frequency per day. Black arrow indicates the day of E-tube and EG-tube placement. After tube placement, regurgitation decreased remarkably, and body weight increased steadily. Asterisks indicate the days of E-tube clogging and replacements performed. 
made of polyvinyl chloride hardened by gastric acid, material of the tubes were changed to silicon in second and third E-tubes. Hardening of the second and third tubes was not observed at tube replacement and autopsy. Therefore, it is recommend to select the tube made of silicon or polyurethane that is resistant to gastric acid.

This has also very recently been described in 4 dogs [8]. That study has reported the E-tube might develop esophageal erosions. Thus, the possibility of development of esophageal erosion or ulcer should be considered in patients placing E-tube. Moreover, the dog presented mucosal hyperplasia and mortal gastric ulcer as a result of placement of EG-tube. In this case, we should have changed the EG-tube to the conventional gastrostomy tube when the nutritional state was improved. Since EG-tube was placed trans-cardia, there is a risk for reflux esophagitis. Anti-acidic agent, such as proton pump inhibitor, should be administered when we use EG-tube to the patients. These risks described above should be considered when we use EG-tube for feeding in dogs.

In summary, drainage of esophageal fluid by using the E-tube was effective in decreasing the frequency of either regurgitation and aspiration pneumonia; thus, long-term management of IME was achieved. Although the EG-tube placement is an alternative way of nutritional support for the patients with IME, this procedure has potential risks of complicating gastric ulceration. Further controlled studies are needed to show a benefit of E-tube esophageal drainage in dogs with IME and ongoing regurgitation.

\section{REFERENCES}

1. Arnell, K., Hill, S., Hart, J. and Richter, K. 2013. Persistent regurgitation in four dogs with caudal esophageal neoplasia. J. Am. Anim. Hosp. Assoc. 49: 58-63. [Medline] [CrossRef]

2. Gaynor, A. R., Shofer, F. S. and Washabau, R. J. 1997. Risk factors for acquired megaesophagus in dogs. J. Am. Vet. Med. Assoc. 211: $1406-1412$. [Medline]

3. Harvey, C. E., O'Brien, J. A., Durie, V. R., Miller, D. J. and Veenema, R. 1974. Megaesophagus in the dog: a clinical survey of 79 cases. J. Am. Vet. Med. Assoc. 165: 443-446. [Medline]

4. Jaggy, A., Oliver, J. E., Ferguson, D. C., Mahaffey, E. A. and Glaus, T. Jr. 1994. Neurological manifestations of hypothyroidism: a retrospective study of 29 dogs. J. Vet. Intern. Med. 8: 328-336. [Medline] [CrossRef]

5. Jergens, A. E. 2005. Diseases of the esophagus. pp. 1298-1309. In: Textbook of Veterinary Internal Medicine. 6th ed., Saunders Elsevier, Philadelphia.

6. Johnson, B. M., Mears, E. A. and DeNovo, R. C. 2009. Canine megaesophagus. pp. 486-492. In: Kirk's Current Veterinary Therapy. 14th ed., Saunders Elsevier, St. Louis.

7. Manning, K., Birkenheuer, A. J., Briley, J., Montgomery, S. A., Harris, J., Vanone, S. L. and Gookin, J. L. 2016. Intermittent At-Home Suctioning of Esophageal Content for Prevention of Recurrent Aspiration Pneumonia in 4 Dogs with Megaesophagus. J. Vet. Intern. Med. 30: $1715-1719$. [Medline] [CrossRef]

8. McBrearty, A. R., Ramsey, I. K., Courcier, E. A., Mellor, D. J. and Bell, R. 2011. Clinical factors associated with death before discharge and overall survival time in dogs with generalized megaesophagus. J. Am. Vet. Med. Assoc. 238: 1622-1628. [Medline] [CrossRef]

9. Niessen, S. J., Eastwood, J., Smyth, J. B. and Cherubini, G. B. 2007. Five cases of canine dysautonomia in England (2004 to 2006). J. Small Anim. Pract. 48: 346-352. [Medline] [CrossRef]

10. Peterson, M. E., Kintzer, P. P. and Kass, P. H. 1996. Pretreatment clinical and laboratory findings in dogs with hypoadrenocorticism: 225 cases (1979-1993). J. Am. Vet. Med. Assoc. 208: 85-91. [Medline]

11. Shelton, G. D., Willard, M. D., Cardinet, G. H. 3rd. and Lindstrom, J. 1990. Acquired myasthenia gravis. Selective involvement of esophageal, pharyngeal, and facial muscles. J. Vet. Intern. Med. 4: 281-284. [Medline] [CrossRef]

12. Simpson, J. 1994. Management of megaosophagus in the dog. In Pract. 16: 14-16. [CrossRef]

13. Washabau, R. J. 2003. Gastrointestinal motility disorders and gastrointestinal prokinetic therapy. Vet. Clin. North Am. Small Anim. Pract. 33: 1007-1028, vi. [Medline] [CrossRef]

14. Willard, M. D. 2003. Disorders of the oral cavity, pharynx, and esophagus. pp.410-412. In: Small Animal Internal Medicine.3rd ed., Mosby, St. Louis.

15. Wray, J. D. and Sparkes, A. H. 2006. Use of radiographic measurements in distinguishing myasthenia gravis from other causes of canine megaoesophagus. J. Small Anim. Pract. 47: 256-263. [Medline] [CrossRef] 